\title{
Electron Correlations in Partially Filled Lowest and Excited Landau Levels
}

\author{
Arkadiusz Wójs \\ Department of Physics, University of Tennessee, Knoxville, Tennessee 37996 \\ Institute of Physics, Wroclaw University of Technology, Wroclaw 50-370, Poland
}

\begin{abstract}
The electron correlations near the half-filling of the lowest and excited Landau levels (LL's) are studied using numerical diagonalization. It is shown that in the low lying states electrons avoid pair states with relative angular momenta $\mathcal{R}$ corresponding to positive anharmonicity of the interaction pseudopotential $V(\mathcal{R})$. In the lowest LL, the super-harmonic behavior of $V(\mathcal{R})$ causes Laughlin correlations (avoiding pairs with $\mathcal{R}=1$ ) and the Laughlin-Jain series of incompressible ground states. In the first excited LL, $V(\mathcal{R})$ is harmonic at short range and a different series of incompressible states results. Similar correlations occur in the paired Moore-Read $\nu=\frac{5}{2}$ state and in the $\nu=\frac{7}{3}$ and $\frac{8}{3}$ states, all having small total parentage from $\mathcal{R}=1$ and 3 and large parentage from $\mathcal{R}=5$. The $\nu=\frac{7}{3}$ and $\frac{8}{3}$ states are different from Laughlin $\nu=\frac{1}{3}$ and $\frac{2}{3}$ states and, in finite systems, occur at a different LL degeneracy (flux). The series of Laughlin correlated states of electron pairs at $\nu=2+2 /\left(q_{2}+2\right)=\frac{8}{3}, \frac{5}{2}, \frac{12}{5}$, and $\frac{7}{3}$ is proposed, although only in the $\nu=\frac{5}{2}$ state pairing has been confirmed numerically. In the second excited LL, $V(\mathcal{R})$ is sub-harmonic at short range and (near the half-filling) the electrons group into spatially separated larger $\nu=1$ droplets to minimize the number of strongly repulsive pair states at $\mathcal{R}=3$ and 5 .

71.10.Pm, 73.20.Dx, 73.40.Hm
\end{abstract}

\section{INTRODUCTION}

When a pure two-dimensional electron gas (2DEG) in a high magnetic field fills a fraction $\nu$ of a degenerate Landau level (LL), the nature of the ground state (GS) and low lying excitations are completely determined by their (Coulomb) interaction. The correlations induced by this interaction can be probed in transport or optical measurements, and, for example, the occuprence of non-degenerate incompressible liquid-like GS's at certain values of $\nu$ is responsible for the fractional quantum Hall $(\mathrm{FQH})$ effect. 6 In the lowest $(n=0) \mathrm{LL}$, the FQH effect is observed at various filling factors $\nu=\frac{1}{3}, \frac{2}{3}, \frac{2}{5}$ etc., all being simple odd-denominator fractions. The origin of these fraftions lies in the special form of (Laughlin) correlations which result from the short-range sharacter of the Coulomb interaction pseudopotential $\mathrm{H}_{\mathrm{H}}$ in the lowest LL. The explanation of all the observed fractions involves identification of Laughlin incompressible GS's at $\nu=(2 p+1)^{-1}$, where $p$ is an ipteger, and their elementary (quasiparticle) excitations, 1 and the observation that at certain fillings $\nu_{\mathrm{QP}}$ the quasiparticles form Laughlin incompressible GS's of their own.8 10 This (Haldane's) hierarchy construction predicts no incompressible GS's at even-denominator fractions, in perfect agreement with the experiments in the lowest LL. Because of its equivalence 11.12 to Haldane's hierarchy picture, Jain's non-interacting composite fermion (CF) modele 16 also predicts FQH states at the same fractions.

Quite surprisingly, the FQH effect at an evendenominator fraction has been discovered 121 in the half-filled first excited $(n=1)$ LL. The incompressibility at $\nu=2+\frac{1}{2}=\frac{5}{2}$ could not be explained within Haldane's hierarchy (or Jain's non-interacting CF) picture and it was immediately obvious that it implied a different type of correlations. Since even-denominator Laughlin states occur for bosons, electron pairing was suggested by Halperin, 25 and various explicit paired-state trial wavef futions have been constructed by a number of authors. 2628 Although earlier theories 53 suggested $s$-pairing (spin depolarization due to a small Zeeman energy; an idea later seemingly supported by experiments in tilted magnetic field 19 21), it is now established22, 29 that the $\nu=\frac{5}{2}$ state is well described by a spin-polarized wavefunction introduced by Moere and Read (MR).27 Mor 22 and Rezayi and Haldane 29 compared the actual Coulomb eigenstates of up to 16 electrons with different trial wavefunctions, and found that the $\nu=\frac{5}{2}$ GS has large overlap with the (particle-hole symmetrized) MR27 state, the phase transition between the "CF behavior" and pairing is driven by the strength of interaction at short range, and the actual Coulomb pseudopotential in the $n=1 \mathrm{LL}$ is close to the transition point.

While the non-Laughlin character of the $\nu=\frac{5}{2}$ state follows from Haldane's "odd-denominator" rule, the type of correlations that cause incompressibility of other FQH states observed 721 in the $n=1$ LL have not yet been completely understood. The occurrence of the FQH effect at such prominent Laughlin-Jain fractions as $\nu=$ $2+\frac{1}{3}=\frac{7}{3}, 2+\frac{2}{3}=\frac{8}{3}$, or $2+\frac{1}{5}=\frac{11}{5}$ might indicate that, although weakened because of reduction of Coulomb repulsion at short range, Laughlin correlations persist in the excited $(n=1)$ LL. The decrease of excitation gaps (e.g., the gap at $\nu=\frac{7}{3}$ being smaller than at $\nu=\frac{1}{3}$ ) could be interpreted as a direct measure of this weakening, and it might seem natural that only the most prominent FQH states of the $n=0$ LL persist at $n=1$. Consequently, one could try to model correlations in the excited LL's using some modified version of the hierarchyer CF picture. For example, it has been proposed 23, 4 that the 
CF's are formed in excited LL as well (i.e., the electrons bind vortices of the many-body wavefunction - which is a definition of Laughlin correlations), although the effects of $\mathrm{CF}-\mathrm{CF}$ interaction (pairing) are more important at $n=1$. On the other hand, numerical calculations 32 ,6 seem to disagree with experiments by showing neither Laughlin correlations nor incompressibility at $\nu=\frac{7}{3}$ For example, quite different energy spectra are obtained 6 for $N \leq 11$ electrons at the same value of the LL degeneracy (flux) corresponding to the Laughlin $\nu=\frac{1}{3}$ filling of the $n=0$ and $n=1$ LL's. In the $n=1 \mathrm{LL}$, the Laughlin quasiparticles or the magneto-roton band do not occur, and the excitation gap oscillates as a function of $N$ and does not converge to a finite value for $N \rightarrow \infty$.

The occurrence of an incompressible GS at a specific filling factor results from the type of correlations that generally occur in the low lying states near this filling. Therefore, these correlations must be studied before the correct trial wavefunctions can be constructed (or, at least, before their success can be understood). The correlations near the half-filling of the lowest and excited LL's are the main subject of this paper. We assume complete spin-polarization of the partially filled LL and perform the numerical calculations in Haldane's spherical geometry, where each LL has the form of a $(2 l+1)$ fold degenerate angular momentum shell. The correlations in a Hilbert space restricted to an isolated LL are best defined through the occupation numbers (fractional parentage by the relative pair angular momentum $\mathcal{R}$. The $\mathcal{G}(\mathcal{R})$ contains more information about the nature of a studied many-body state than its overlap with a trial wavefunction. It is also easier to interpret than the real-space pair-correlation function $g(r)$.

We explain the effects of harmonic $\left(V_{\mathrm{H}}\right)$ and anharmonic $\left(V_{\mathrm{AH}}\right)$ parts of the interaction pseudopotential $V=V_{\mathrm{H}}+V_{\mathrm{AH}}$ on correlations, and formulate a simple theorem which links the $\mathcal{G}(\mathcal{R})$ profile of low lying states with the sign of $V_{\mathrm{AH}}(\mathcal{R})$. The Laughlin correlations occur when $V_{\mathrm{AH}}(\mathcal{R})>0$ and disappear when $V(\mathcal{R})$ becomes harmonic at short range. 0 This clarifies the physical meaning of the critical strength of the highest pseudopotential parameter (relative to the Coulomb value) at which the transition hetween the Laughlin and MR phases has been found.22 29

From the analysis of the energy spectra of $N \leq 16$ electrons at different values of $2 l$ (LL degeneracy), we identify three series of non-degenerate $(L=0)$ GS's which in the thermodynamic limit of $N \rightarrow \infty$ and $N /(2 l+1) \rightarrow \nu$ converge to the incompressible states at $\nu=\frac{5}{2}, \frac{7}{3}$, and $\frac{8}{3}$. As shown by Morf.22 the finite-size MR $\nu=\frac{5}{2}$ states occur for even $N$ at $2 l=2 N+1$. The $\nu=\frac{7}{3}$ state occurs at $2 l=3 N-7$, which is different than $2 l=3 N-3$ of the Laughlin $\nu=\frac{1}{3}$ state (the same is true for their particle-hole symmetric conjugates at $\nu=\frac{8}{3}$ and $\frac{2}{3}$ ).

The analysis of the $\mathcal{G}(\mathcal{R})$ curves obtained for different values of $N$ and $2 l$ and different model pseudopotentials shows that the electron correlations near the half-filling of the $n=1$ LL depend critically on the harmonic behavior of $V(\mathcal{R})$ at short range. (at $\nu \leq \frac{9}{4}$ the CF picture with four attached fluxes works and for example the $\nu=\frac{11}{5}$

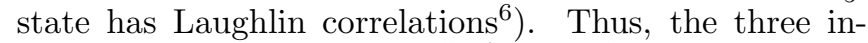
compressible states at $\nu=\frac{5}{2}, \frac{7}{3}$, and $\frac{8}{3}$ all have similar (not Laughlin electron-electron, although maybe Laughlin pair-pair) correlations. In all low lying states near the half-filling, electrons minimize the total parentage from two pair states of highest repulsion, $\mathcal{R}=1$ and 3 , which results in $\mathcal{G}(1) \approx \mathcal{G}(3)$ and large value of $\mathcal{G}(5)$. Cusps in the dependence of $\mathcal{G}(1)+\mathcal{G}(3)$ and $\mathcal{G}(5)$ on $N$ and $2 l$ coincide with occurrence of incompressible $\nu=\frac{5}{2}, \frac{7}{3}$, and $\frac{8}{3}$ states (similar to cusps in $\mathcal{G}(1)$ and $\mathcal{G}(3)$ in the $n=0 \mathrm{LL}$ signalling the Laughlin-Jain states). For the $\operatorname{MR} \nu=\frac{5}{2}$ state, the number of $\mathcal{R}=1$ pairs is roughly equal to the half of the electron number, $\frac{1}{2} N$, which supports the conjecture of pairing.

In the second excited $(n=2)$ LL, $V(\mathcal{R})$ is subharmonic at short range and super-harmonic at long range, and the minimization of energy requires avoidance of strongly repulsive pair states at the intermediate $\mathcal{R}=3$ and 5 , that is having $\mathcal{G}(3) \approx \mathcal{G}(5)<\mathcal{G}(1) \approx \mathcal{G}(7)$. This is achieved by grouping of electrons into spatially separated $\nu=1$ droplets. Our values of $\mathcal{G}(1)$ suggest that in a finite system each droplet consists of three electrons. This precludes pairing in the $\nu=\frac{9}{2}$ state, but not formation of larger droplets or the charge-density-wave stripe order 33.34 in an infinite system.

\section{MODEL}

We consider a system of $N$ electrons confined on a Haldane sphere 8 of radius $R$. The magnetic field $B$ normal to the surface is produced by a Dirac magnetic monopole placed at the origin. The strength $2 S$ of the monopole is defined in the units of flux quantum $\phi_{0}=h c / e$, so that $4 \pi R^{2} B=2 S \phi_{0}$ and the magnetic length is $\lambda=R / \sqrt{S}$ The single-particle states (monopole harmonics) 8, 35, 36 are the eigenstates of angular momentum $l \geq S$ and its projection $m$. The single-particle energies fall into $(2 l+1)$-fold degenerate angular momentum shells (LL's), and the $n$-th shell has $l=S+n$.

At large $B$, the electron-electron (Coulomb) interaction is weak compared to the cyclotron energy $\hbar \omega_{c}$, and the scattering between different LL's can be neglected. In the low lying many-electron states at a filling factor $\nu_{\text {tot }}=2 f+\nu$ (where $f$ is an integer and $\left.\nu<1\right)$, a number $f$ of lowest LL's (with $n=0,1, \ldots, f-1$ ) are completely filled. For simplicity, in the following we will omit the subscript 'tot' and, depending on the context, $\nu$ will denote either partial filling of the highest occupied LL or the total filling factor $\nu_{\text {tot }}$.

The Coulomb interaction within a partially filled LL (with $n=f$ ) is given by a pseudopotential $50 V_{\mathrm{C}}^{(n)}(\mathcal{R})$. The pseudopotential $V(\mathcal{R})$ is defined as the interaction 
energy $V$ of a pair of particles as a function of their relative angular momentum $\mathcal{R}$. On a sphere, $\mathcal{R}=2 l-L^{\prime}$ where $L^{\prime}=\left|\mathbf{l}_{1}+\mathbf{l}_{2}\right|$ is the total pair angular momentum. For identical (spin-polarized) fermions, $\mathcal{R}$ is an odpinteger, and larger $\mathcal{R}$ means larger average separation. 6

The many-electron Hamiltonian can be written as

$$
H=\sum_{i j k l} c_{i}^{\dagger} c_{j}^{\dagger} c_{k} c_{l}\langle i j|V| k l\rangle+\mathrm{const}
$$

where $c_{m}^{\dagger}\left(c_{m}\right)$ creates (annihilates) an electron in state $|l=S+f, m\rangle$ of the $n=f$ LL, the two body interaction matrix elements $\langle i j|V| k l\rangle$ are related with $V(\mathcal{R})$ through the Clebsch-Gordan coefficients. The constant term includes the energy of the completely filled LL's with $n<f$, the cyclotron energy of the electrons in the $n=f$ LL, and their interaction with the underlying (rigid) completely filled LL's, and will be omitted.

Hamiltonian (1) is diagonalized numerically in Haldane's spherical geometry, for a finite number $N$ of electrons at different values of $2 l$, corresponding to $\frac{1}{3} \leq \nu \frac{2}{3}$. The result is the spectrum of energy $E$ as a function of total angular momentum $L$. The $L=0$ ground states (GS) separated from the rest of the spectrum by an excitation gap $\Delta$ represent the non-degenerate $(k=0)$ GS's an a plane. If a series of such GS's can be identified at increasing $N$ and $2 l=\nu^{-1} N+$ const, and if the gap $\Delta$ does not collapse in the $N \rightarrow \infty$ limit, these GS's describe an incompressible state of an infinite 2DEG at a filling factor $2 f+\nu$.

\section{FRACTIONAL PARENTAGE}

The electric conductivity and other properties that involve electron scattering depend critically on the correlations in the partially filled LL, which in turn depend entirely on the form of interaction pseudopotential $V(\mathcal{R})$. The correlations are best described in terms of the $e_{-}$ efficients of fractional (grand)parentage (CFGP) 6. $60.30,31$ $\mathcal{G}(\mathcal{R})$. The CFGP gives a fraction of electron pairs that are in the pair eigenstate of a given $\mathcal{R}$, and thus $\mathcal{G}(\mathcal{R})$ can be regarded as a pair-correlation function. The energy $E_{L \alpha}$ of a state $|L \alpha\rangle$ can be conveniently expressed through CFGP's as

$$
E_{L \alpha}=\frac{1}{2} N(N-1) \sum_{\mathcal{R}} \mathcal{G}_{L \alpha}(\mathcal{R}) V(\mathcal{R}),
$$

and the normalizaton condition is $\sum_{\mathcal{R}} \mathcal{C}_{\text {the }}{ }^{\alpha}(\mathcal{R})=1$. The CFGP's also satisfy another constraint, 6.0

$$
\begin{aligned}
\frac{1}{2} N(N-1) & \sum_{\mathcal{R}} \mathcal{G}_{L \alpha}(\mathcal{R}) L^{\prime}\left(L^{\prime}+1\right) \\
& =L(L+1)+N(N-2) l(l+1),
\end{aligned}
$$

where $L^{\prime}=2 l-\mathcal{R}$.

\section{LAUGHLIN CORRELATIONS}

The pseudopotential $V_{\mathrm{H}}(\mathcal{R})$ of the harmonic interaction $V_{\mathrm{H}}(r) \propto r^{2}$ within an isolated $(n$ th) LL is linear in $L^{\prime}\left(L^{\prime}+1\right), 6$ and from Eqs. (2-3) it follows that its energy spectrum is degenerate at each value of $L$. In other words, the harmonic interaction (within an isolated LL) does not cause any correlations, which are hence entirely determined by the anharmonic part $V_{\mathrm{AH}}(\mathcal{R})$ of the total pseudopotential $V(\mathcal{R})=V_{\mathrm{H}}(\mathcal{R})+V_{\mathrm{AH}}(\mathcal{R})$. Moreover, at a filling factor $\nu \geq(2 p+1)^{-1}$, most important is the behavior of $V(\mathcal{R})$ at $\mathcal{R} \leq 2 p+1$ (corresponding to the pair of "nearest" electrons in the Laughlin state) and at those values where $V(\mathcal{R})$ changes most quickly (i.e. where the "effective force" $\sim \mathrm{d} V / \mathrm{d}\langle r\rangle$ is the largest). The occurrence of Laughlin correlations in the FQH systems and their insensitivity to the details of the pseudopotential result from the following:6.

Theorem 1: If for any three pair states at $\mathcal{R}_{1}<\mathcal{R}_{2}<$ $\mathcal{R}_{3}$ (i.e., at $L_{1}^{\prime}>L_{2}^{\prime}>L_{3}^{\prime}$ ) the pseudopotential $V$ is super-harmonic (i.e., increases more quickly than linearly as a function of $L^{\prime}\left(L^{\prime}+1\right)$; i.e., $V_{\mathrm{H}}\left(\mathcal{R}_{1}\right)=V_{\mathrm{H}}\left(\mathcal{R}_{3}\right)=0$ and $\left.V_{\mathrm{H}}\left(\mathcal{R}_{2}\right)<0\right)$, then the many-electron energy $E_{L}$ can be lowered (within a given Hilbert space $[N, 2 l, L]$ ) by transferring some of the parentage from $\mathcal{G}\left(\mathcal{R}_{1}\right)$ and $\mathcal{G}\left(\mathcal{R}_{5}\right)$ to $\mathcal{G}\left(\mathcal{R}_{3}\right)$ without violating Eq. (3). The same holds in the planar geometry, except that the harmonic pseudopotential on a plane is linear in $\mathcal{R}$.

As a result, if $V(\mathcal{R})$ is a super-harmonic at small $\mathcal{R}$ (at short range), the lowest energy states at each $L$ have minimum possible parentage from the (most strongly repulsive) pair state at the smallest $\mathcal{R}$. The complete avoidance of $p$ pair states at $\mathcal{R}<2 p+1$ corresponds to a Jastrow $\prod_{i<j}\left(z_{i}-z_{j}\right)^{2 p}$ in the many-electron wavefunction and, in particular, the Laughlin incompressible $\nu=(2 p+1)^{-1}$ GSL is the only state at a given $N$ and $2 l$ for which $\mathcal{G}(1)=0$.

\section{PAIRING AND LAUGHLIN PAIRED STATES}

If the pseudopotential is sub-harmonic at small $\mathcal{R}$ (i.e., at short range), for example $V_{\mathrm{H}}(1)=V_{\mathrm{H}}(5)=0$ and $V_{\mathrm{H}}(3)>0$, then it should be energetically favorable to minimize parentage from the $\mathcal{R}=3$ state, even at the cost of a large value of $\mathcal{G}(1)$. Although the resulting $\mathcal{R}=1$ pairs are not formed because of any electronelectron attraction, but rather because of repulsion from the surrounding 2DEG (and thus their stability depends on $\nu$ ), the many-electron correlations can be described in terms of electron pairing and the (possibly simpler) correlations between pairs. On a sphere, each $\mathcal{R}=1$ pair is a boson with the total angular momentum of $l_{2}=2 l-1$. The two-boson pair states are labeled by the total angular momentum $L_{2}^{\prime}=2 l_{2}-\mathcal{R}_{2}$ where $\mathcal{R}_{2}$ is an even integer, and the pair-pair interaction is defined by an effective pseudopotential $V_{2}\left(\mathcal{R}_{2}\right)$. The Pauli exclusion principle 
applied to individual electrons results in a hard core at a number $p_{2}=2 \mathrm{gf}$-lowest values of $\mathcal{R}_{2}$ (similar to that of charged excitons 37 ), so that $\mathcal{R}_{2} \geq 2 p_{2}$ for all pairs. Such hard core can be accounted for by a mean field (MF) composite boson (CB) transformation with $2 p_{2}$ flux quanta attached to each boson. The CB transformation gives an effective CB angular momentum $l_{2}^{*}=l_{2}-p_{2}\left(N_{2}-1\right)$, where $N_{2}$ is the number of pairs. In the CB picture, all many-boson $L$-multiplets can be obtained by addition of $N_{2}$ angular momenta $l_{2}^{*}$ of individual CB's (without an additional hard core). For example, the $\nu=1$ state of electrons corresponds to the condensate of CB's in their only available $l_{2}^{*}=0$ state. If the pair-pair pseudopotential $V_{2}\left(\mathcal{R}_{2}\right)$ is super-harmonic (and $l_{2}^{*}>0$ ), an additional MF CB transformation attaching an even number of $2 q_{2}$ fluxes to each pair can be applied to select the lowest energy band of paired states which avoid a number of $q_{2}$ lowest values of $\mathcal{R}_{2}$ beyond the hard core. The electron and CB filling factors, in the $N \rightarrow \infty$ limit defined as $\nu=N / 2 l$ and $\nu_{2}^{*}=N_{2} / 2 l_{2}^{*}$, are related by

$$
\nu^{-1}=\left(4 \nu_{2}^{*}\right)^{-1}+1
$$

and, for example, the series of Laughlin correlated CB states at $\nu_{2}^{*}=\frac{1}{8}, \frac{1}{6}, \frac{1}{4}$, and $\frac{1}{2}$ occur at the electron filling factors $\nu=\frac{1}{3}, \frac{2}{5}, \frac{1}{2}$, and $\frac{2}{3}$, respectively. It is quite remarkable that, coincidentally, some of the most prominent odd-denominator Laughlin-Jain fractions occur among these states along with the (evendenominator) half-filled state.

On a Haldane's sphere, Laughlin $\nu_{2}^{*}=\left(2 q_{2}\right)^{-1}$ states of bosons have $2 l_{2}^{*}=2 q_{2}\left(N_{2}-1\right)$, and thus the Laughlincorrelated paired $\nu=2 /\left(q_{2}+2\right)$ states occur at

$$
2 l=\frac{q_{2}+2}{2} N-1-q_{2} .
$$

It is noteworthy that applying the particle-hole symmetry $\left(N \leftrightarrow N_{h}\right.$, where $N_{h}=2 l+1-N$ is the number of holes in the isolated LL) to Eq. (5) generates a different series of states at

$$
2 l=\frac{q_{2}+2}{q_{2}} N+1
$$

That is because (in a finite system on a sphere) Laughlin paired states of electrons at $\nu$ do not occur at the same values of $2 l$ as the Laughlin paired states of holes at $1-\nu$ (a similar effect was discussed in Ref. 38).

If only a fraction $2 N_{2} / N<1$ of electrons formed pairs in a many-electron state, the correlations should be described in terms of $N_{2}$ pairs (bosons) and $N_{1}=$ $N-2 N_{2}$ excess electrons (fermions). The pair states of one electron and one electron pair are labeled by $L_{12}^{\prime}=$ $l_{1}+l_{2}-\mathcal{R}_{12}$ where $l_{1} \equiv l$ and $\mathcal{R}_{12}$ is any integer, and the electron-pair interaction is defined by $V_{12}\left(\mathcal{R}_{12}\right)$. A multi-component MF composite particle (CP) transformation can be used to account for the electron-pair hard core which forbids $\mathcal{R}_{12}<2$. In such transformation, 37

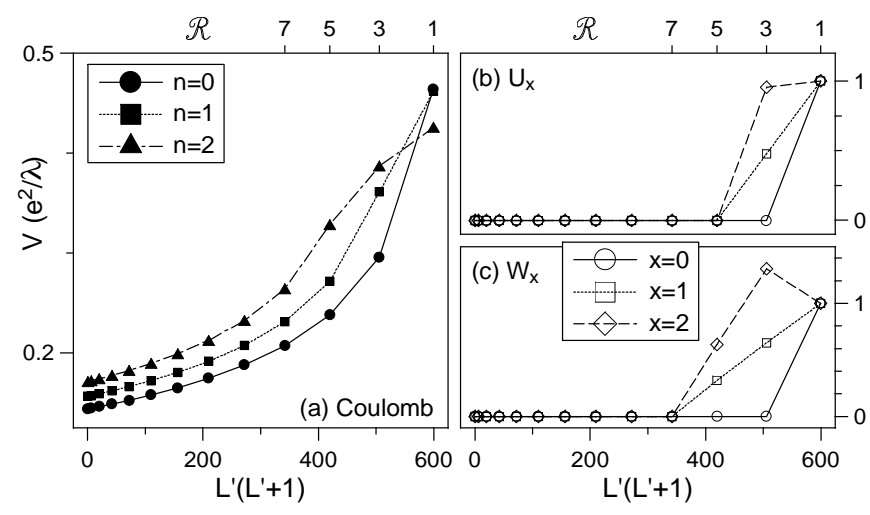

FIG. 1. The pseudopotentials (energy vs. squared pair angular momentum) of the Coulomb interaction $V_{\mathrm{C}}^{(n)}$ in the lowest $(n=0)$ and two excited $(n=1$ and 2$)$ Landau levels (a), and of the model interactions $U_{x}$ (b) and $W_{x}$ (c), calculated for Haldane's sphere with $2 l=25 . \quad \lambda$ is the magnetic length.

each electron couples to $p_{12}=2$ flux quanta attached to each pair, and each pair sees equal number $p_{12}$ of fluxes attached to each electron (in addition to $2 p_{2}$ fluxes that each pair sees on every other pair), giving $\mathrm{CF}$ and $\mathrm{CB}$ angular momenta $l_{2}^{*}=l_{2}-\frac{1}{2} p_{12} N_{1}-p_{2}\left(N_{2}-1\right)$ and $l_{1}^{*}=l_{1}-\frac{1}{2} p_{12} N_{2}$. It is easy to check that a full shell of $N=2 l+1$ electrons (the $\nu=1$ state) can be viewed as the only available state of $N_{2}$ pairs and $N_{1}=2 l+1-2 N_{2}$ excess electrons, in which the pairs condense at $l_{2}^{*}=0$ and the electrons completely fill their CF shell of $2 l_{1}^{*}=N_{1}-1$.

If both electron-pair and pair-pair repulsions are super-harmonic, additional CP transformations can be used to select low energy states in which an appropriate number of electron-electron, pair-pair, and electron-pair pair states at the smallest $\mathcal{R}_{1}, \mathcal{R}_{2}$, and $\mathcal{R}_{12}$, respectively, are avoided. While the discussion of the multi-component electron-pair (boson-fermion) liquids with Laughlin correlations will be presented elsewhere, 39 let us note that such a state might be a more appropriate description of the $\nu=\frac{7}{3}$ state than a fully paired $\nu_{2}^{*}=\frac{1}{8}$ state.

The idea of a paired incompressible GS at $\nu=\frac{5}{2}$ (half-filled -1 LL) has been suggested by a number of authors 12528 as the even-denominator fractions are characteristic of Laughlin-correlated systems of bosons. However, as shown in Fig. 17(a), the Coulomb pseudopotential $V_{\mathrm{C}}^{(1)}(\mathcal{R})$ in the first excited LL is almost harmonic (linear in $L^{\prime}\left(L^{\prime}+1\right)$ ) rather than sub-harmonic between $\mathcal{R}=1$ and 5 , and super-harmonic at larger $\mathcal{R}$. Whether the above-sketched CP picture correctly describes correlations in the $\nu=\frac{5}{2}$ state depends on whether the harmonicity (or weak super-harmonicity) of $V_{\mathrm{C}}^{(1)}(\mathcal{R})$ at $\mathcal{R} \leq 5$ is sufficient to cause pairing. If only the pairs are formed, the pair-pair repulsion will certainly be superharmonic (for the relevant $\mathcal{R}_{2}$ ) because the Coulomb repulsion in the $n=1$ LL is sub-harmonic only for small 
$\mathcal{R}$, and not for electrons that belong to different pairs.

It is noteworthy that inclusion of the effects of the finite width of the quasi-2D electron layer even enhances the harmonicity of the Coulomb pseudopotential at short range. This is because the pseudopotential of the $3 \mathrm{D}$ Coulomb interaction $V(r, z) \propto 1 / \sqrt{r^{2}+z^{2}}$ in a quasi-2D layer of width $w$ can be well approximated by that of an effective 2D potential $V(r) \propto 1 / \sqrt{r^{2}+d^{2}}$ with $d=w / 5$, and because $V(r) \approx\left(1-r^{2} / 2 d^{2}\right) / d$ at small $r$. One can expect that other effects (such as due to the LL mixing) are too weak to produce large anharmonicity, and thus that the actual pseudopotential that occurs in the experimental systems is indeed nearly harmonic at $\mathcal{R} \leq 5$.

\section{NUMERICAL ENERGY SPECTRA FOR THE COULOMB PSEUDOPOTENTIAL}

If an incompressible GS occurs in an infinite system at a certain filling factor $\nu$, and if the correlations responsible for the incompressibility have a finite (short) range $\xi$, then the $L=0$ (non-degenerate) GS's are expected to occur in sufficiently large $(R>\xi)$ finite (spherical) systems for a series of electron numbers $N$ and LL degeneracies $2 l+1$, such that $N /(2 l+1) \rightarrow \nu$ for $N \rightarrow \infty$. In particular, for the $\nu=\frac{1}{2}$ filling (of the $n=1 \mathrm{LL}$; relevant for the $\nu=\frac{5}{2}$ state) we expect such series at $N /(2 l+1) \rightarrow \frac{1}{2}$, for which $N_{h} / N \rightarrow 1$. The excitation gaps $\Delta$ above the $L=0$ GS's are generally expected to decrease as a function of $N$ (as the size quantization weakens) but it must converge to a finite value $\Delta_{\infty}>0$ in the $N \rightarrow \infty$ limit.

We have calculated the energy spectra of up to 16 electrons filling $\frac{1}{3} \leq \nu \leq \frac{2}{3}$ of the lowest, first excited, and second excited LL. Due to the particle-hole symmetry in an isolated LL $\left(N \leftrightarrow N_{h}\right)$, only the systems with $N_{h} \geq N$ need be considered. The dependence of the GS degeneracy and excitation gap $\Delta$ on $N$ and $2 l$ (i.e., on $N$ and $\mu$ ) is different in different LL's. As pointed out by Morf,22 near the half-filling of the $n=1$ LL the non-degenerate $(L=0)$ GS's with the largest excitation gaps occur in systems with the even values of $N$ and $\left|N-N_{h}\right|=2$. This corresponds to even $N$ and $2 l=2 N-3$, the values for the MR $\nu=\frac{5}{2}$ state, or its particle-hole conjugate at $2 l=2 N+1$. Indeed, these numerical GS's were shown 22 to have large overlap with the spherical version of the exact MR trial wavefunction. Note also that, as given by Eq. (6), the value $2 l=2 N-3$ describes the Laughlin $\nu_{2}^{*}=\frac{1}{4}$ state of $\mathcal{R}=1$ pairs. The excitation gaps for $N=N_{h}+2=10,12,14$, and 16 electrons are $\Delta=0.0192,0.0258,0.0220$, and $0.0219 e^{2} / \lambda$, respectively. A similar series of non-degenerate $(L=0)$ GS's with slightly smaller gaps occur for all even values of $N=N_{h}$ (i.e., at $2 l=2 N-1$ ), except for $N=10$. Both these series correspond to the half-filled $n=1$ level (i.e. to $\nu=\frac{5}{2}$ ) in the $N \rightarrow \infty$ limit. In the following, we assume that the series of $N$-electron GS's at $2 l=2 N+1$ in the $n=1$ LL describes the $\nu=\frac{5}{2}$ state of an infinite (planar) system, and study correlations in these states.

We have also identified two other series of nondegenerate GS's with fairly large excitation gaps. One series occurs at both odd and even values of $N$ and at $2 l=3 N-7$, and these GS's correspond to the $\nu=\frac{7}{3}$ filling in the $N \rightarrow \infty$ limit. The gaps for $N=8,9, \ldots$, 12 electrons are $\Delta=0.0192,0.0295,0.0217,0.0140$, and $0.0049 e^{2} / \lambda$, respectively. From the particle-hole symmetry, the other, conjugate $\left(\nu=\frac{8}{3}\right)$ series occurs at even values of $N$ and at $2 l=\frac{3}{2} N+2$. Note that neither of these series occur at the values of $2 l$ given by Eqs. (5) or (6) corresponding to the Laughlin paired $\nu_{2}^{*}=\frac{1}{8}$ (for $\nu=\frac{7}{3}$ ) or $\nu_{2}^{*}=\frac{1}{2}\left(\right.$ for $\left.\nu=\frac{8}{3}\right)$ state.

\section{NUMERICAL ENERGY SPECTRA FOR MODEL PSEUDOPOTENTIALS}

The pseudopotential of the Coulomb $\left(\propto r^{-1}\right)$ interaction is different in different LL's. For $n=0$ it is superharmonic in the entire range of $\mathcal{R}$, while for $n=1$ it is super-harmonic at $\mathcal{R} \geq 5$ but harmonic between $\mathcal{R}=1$ and 5 . To study the transition of the electron system at $\nu \geq \frac{1}{3}$ from the Laughlin- to MR-correlated phase we use a model pseudopotential $U_{x}(\mathcal{R})$ shown in Fig. 1(b), for which $U_{x}(1)=1, U_{x}(\mathcal{R} \geq 5)=0$, and $U_{x}(3)=x \cdot V_{\mathrm{H}}(3)$, where $V_{\mathrm{H}}(3)$ is the "harmonic" value defined so that $U_{1}$ is linear in $L^{\prime}\left(L^{\prime}+1\right)$ for $\mathcal{R}$ between 1 and 5 . The $U_{x}(\mathcal{R})$ is intended to model the anharmonic part of a repulsive (Coulomb) pseudopotential (at short range). The omitted harmonic part does not affect many-electron wavefunctions and only results in a shift of the entire energy spectrum by a constant $\propto L(L+1)$. The variation of $x$ in $U_{x}(\mathcal{R})$ from $x=0$ through $x=1$ up to $x>1$ (super-harmonic, harmonic, and sub-harmonic at small $\mathcal{R}$, respectively) allows calculation of wavefunctions and energy spectra of systems whose low energy states have well known correlations (Laughlin correlations at $x=0$ and pairing or grouping into larger clusters at $x \gg 1$ ), and their comparison with those of Coulomb pseudopotentials for different $n$. The comparison of the $n=1$ Coulomb energy spectra with the spectra of $U_{x}$ with $x=0, \frac{1}{2}, 1,2$, and 5 is shown in Fig. 2 for the systems of $N=8(\mathrm{a}-\mathrm{f}), 10\left(\mathrm{a}^{\prime}-\mathrm{f}^{\prime}\right)$, and 12 electrons $\left(\mathrm{a}^{\prime \prime}-\mathrm{f}^{\prime \prime}\right)$ at $2 l=2 N+1$, in which the MR GS occurs in the $n=1$ LL. The energy scale is not shown on the vertical axes because the graphs are intended to show the structure of low energy spectra rather than the values of energy (the values obtained for the model pseudopotentials scale with $U_{x}(1)$, which we arbitrarily set equal to unity, and should include additional energy due to the neglected harmonic part of the pseudopotential).

In the spectra for $x<1\left(\mathrm{~b}-\mathrm{b}^{\prime \prime}\right.$ and $\left.\mathrm{c}-\mathrm{c}^{\prime \prime}\right)$ the low lying states have Laughlin correlations and can be understood within the CF (or Haldane's hierarchy) picture. For the three systems used in our example, the lowest states are Jain $\nu=\frac{2}{5}$ GS at $L=0$ and the band of excited states 


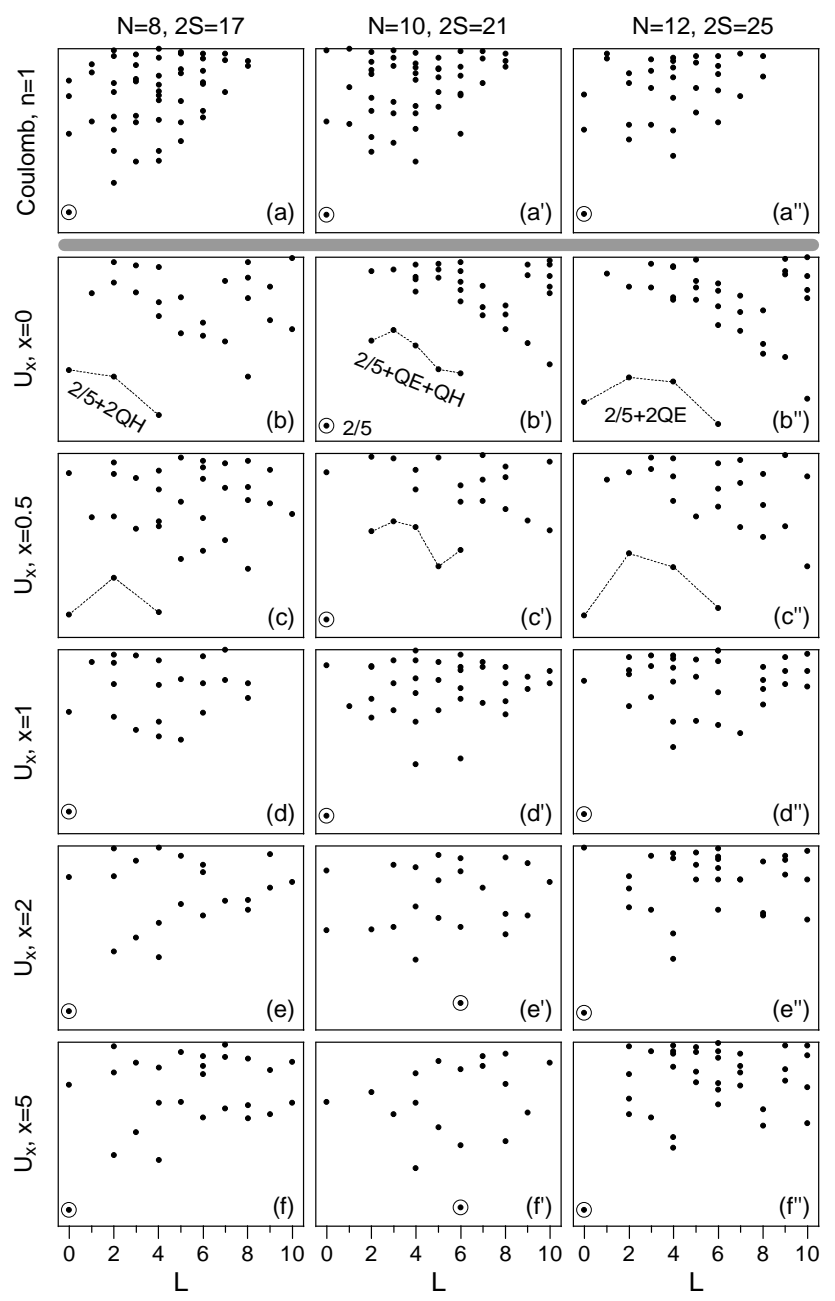

FIG. 2. The $N$-electron energy spectra (energy vs. angular momentum $L$ ) on a Haldane's sphere: $N=8$ and $2 l=17$ $(\mathrm{a}-\mathrm{f}), N=10$ and $2 l=21\left(\mathrm{a}^{\prime}-\mathrm{f}^{\prime}\right)$, and $N=12$ and $2 l=25$ $\left(\mathrm{a}^{\prime \prime}-\mathrm{f}^{\prime \prime}\right)$, calculated for the Coulomb pseudopotential in the first excited Landau level $V_{\mathrm{C}}^{(1)}\left(\mathrm{a}^{-\mathrm{a}^{\prime \prime}}\right)$, and for model interaction $U_{x}$ with $x$ between $0\left(\mathrm{~b}-\mathrm{b}^{\prime \prime}\right)$ and $5\left(\mathrm{f}-\mathrm{f}^{\prime \prime}\right)$. Circles and lines mark the lowest energy states. The Moore-Read $\nu=\frac{5}{2}$ state is the ground state in each Coulomb spectrum.

at $2 \leq L \leq 6$ containing a quasielectron-quasihole (QE$\mathrm{QH})$ pair $\left(\mathrm{b}^{\prime}-\mathrm{c}^{\prime}\right)$, and the states containing a pair of QH's $(\mathrm{b}-\mathrm{c})$ or QE's $\left(\mathrm{b}^{\prime \prime}-\mathrm{c}^{\prime \prime}\right)$ in the $\nu=\frac{2}{5}$ state.

While it is well known that the energy spectra for $x<$ 1 are similar to the Coulomb spectra in the lowest LL, they are clearly different from those in the first excited LL. As expected from the behavior of $V_{\mathrm{C}}^{(1)}(\mathcal{R})$, the best approximation to the $n=1$ Coulomb spectra is obtained for $U_{x}$ with $x \approx 1$. Regardless of the value of GS angular momentum in the $x=0$ spectra, the $L=0$ GS's occur in all the three systems at $x=1$. At $x \gg 1$, when $U_{x}(\mathcal{R})$ becomes strongly sub-harmonic between $\mathcal{R}=1$ and 5 , the $L=0$ GS persists in some systems (f and $\mathrm{f}^{\prime \prime}$ ) but not in others $\left(\mathrm{f}^{\prime}\right)$.

Similar plots for the $\nu=\frac{7}{3}$ spectra of $N=9,10$, and
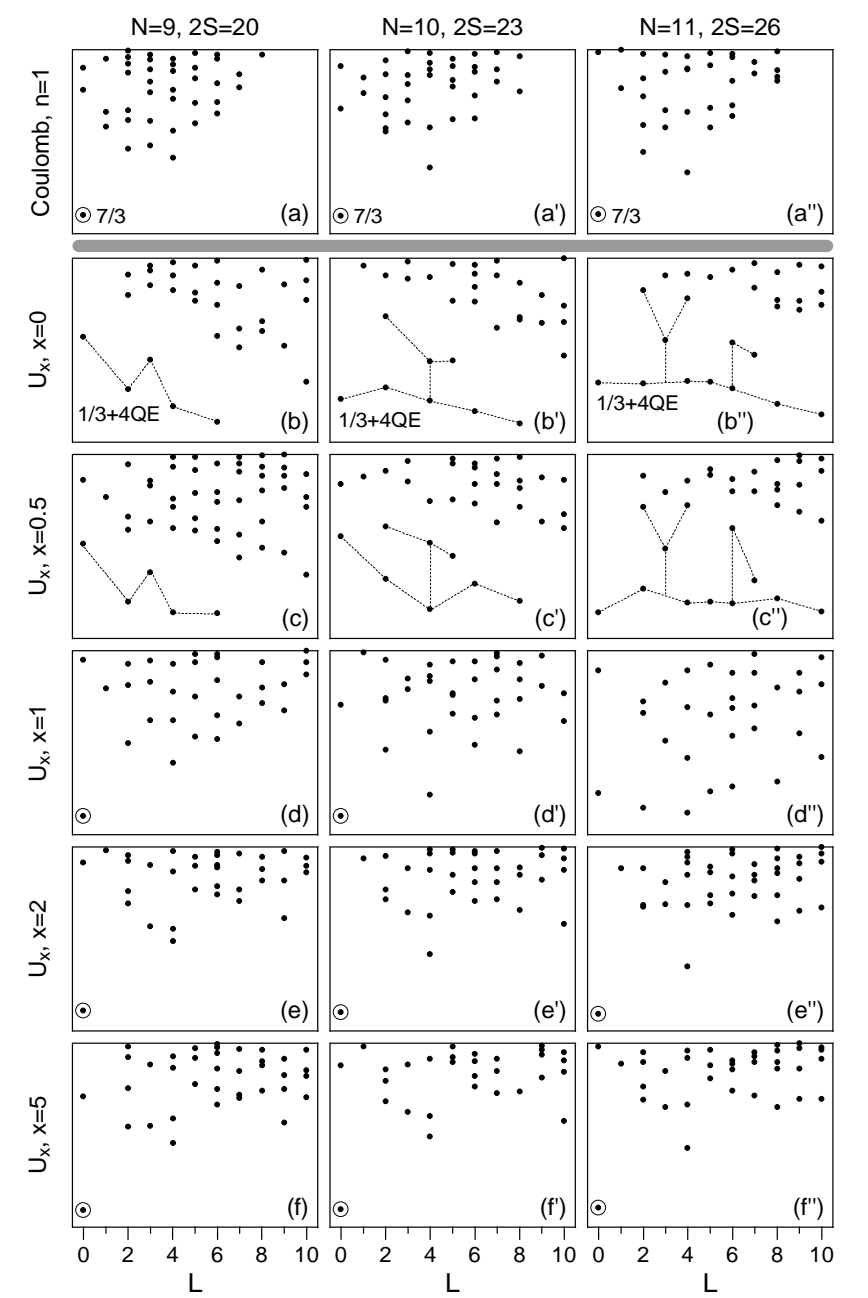

FIG. 3. The $N$-electron energy spectra (energy vs. angular momentum $L$ ) on a Haldane's sphere: $N=9$ and $2 l=20$ $(\mathrm{a}-\mathrm{f}), N=10$ and $2 l=23\left(\mathrm{a}^{\prime}-\mathrm{f}^{\prime}\right)$, and $N=11$ and $2 l=26$ $\left(a^{\prime \prime}-\mathrm{f}^{\prime \prime}\right)$, calculated for the Coulomb pseudopotential in the first excited Landau level $V_{\mathrm{C}}^{(1)}\left(\mathrm{a}-\mathrm{a}^{\prime \prime}\right)$, and for model interaction $U_{x}$ with $x$ between $0\left(\mathrm{~b}-\mathrm{b}^{\prime \prime}\right)$ and $5\left(\mathrm{f}-\mathrm{f}^{\prime \prime}\right)$. Circles and lines mark the lowest energy states. The incompressible $\nu=\frac{7}{3}$ state is the ground state in each Coulomb spectrum.

11 electrons at $2 l=3 N-7$ are shown in Fig. 3. For each $N$, the low lying states of super-harmonic pseudopotentials $U_{0}\left(\mathrm{~b}-\mathrm{b}^{\prime \prime}\right)$ and $U_{0.5}\left(\mathrm{c}-\mathrm{c}^{\prime \prime}\right)$ contain four QE's in the Laughlin $\nu=\frac{1}{3}$ state, while the Coulomb spectra in the $n=1 \mathrm{LL}\left(\mathrm{a}-\mathrm{a}^{\prime \prime}\right)$ all have a $L=0$ ground state with a significant excitation gap, and all resemble the spectra of harmonic and sub-harmonic pseudopotentials $U_{1}\left(\mathrm{~d}-\mathrm{d}^{\prime \prime}\right)$, $U_{2}\left(\mathrm{e}-\mathrm{e}^{\prime \prime}\right)$, and $U_{5}\left(\mathrm{f}-\mathrm{f}^{\prime \prime}\right)$.

\section{CORRELATIONS IN LOW LYING STATES}

To find out if the correlations at $\nu=\frac{5}{2}$ or $\frac{7}{3}$ can be understood in terms of electron pairing, we have analyzed the CFGP's of low lying states near the half filling. In 


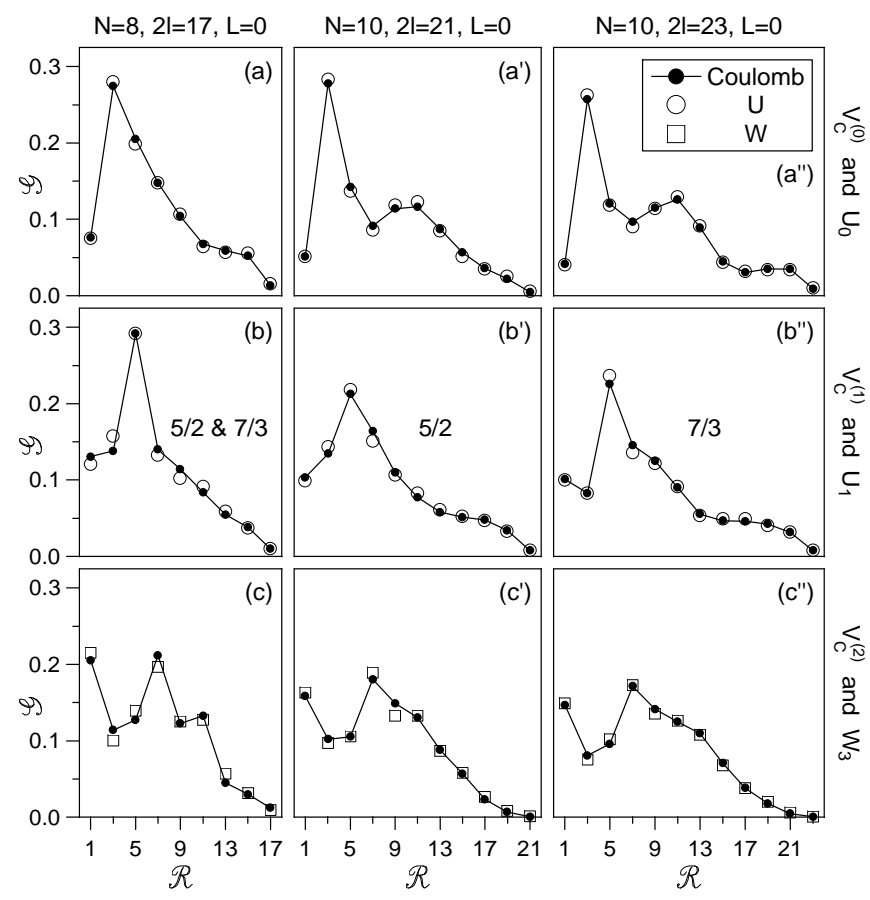

FIG. 4. The pair-correlation functions (coefficient of fractional parentage $\mathcal{G}$ vs. relative pair angular momentum $\mathcal{R}$ ) in the lowest energy $L=0$ state of $N$ electrons on a Haldane's sphere: $N=8$ and $2 l=17(\mathrm{a}-\mathrm{c}), N=10$ and $2 l=21\left(\mathrm{a}^{\prime}-\mathrm{c}^{\prime}\right)$, and $N=10$ and $2 l=23\left(\mathrm{a}^{\prime \prime}-\mathrm{c}^{\prime \prime}\right)$, calculated for the Coulomb pseudopotential in the lowest $\left(\mathrm{a}-\mathrm{a}^{\prime \prime}\right)$, first excited $\left(\mathrm{b}-\mathrm{b}^{\prime \prime}\right)$, and second excited $\left(\mathrm{c}-\mathrm{c}^{\prime \prime}\right)$ Landau level, and for the appropriate model interaction $U_{x}$ or $W_{x}$.

Fig. 1 we show some examples of the full $\mathcal{G}(\mathcal{R})$ profiles (pair-correlation functions) calculated for the lowest $L=$ 0 states of eight and ten electrons at $2 l=2 N+1\left(\nu=\frac{5}{2}\right)$ and $2 l=3 N-7\left(\nu=\frac{7}{3}\right)$. The $N=8$ state at $2 l=17$ (a-c) contains two QH's in the incompressible $\nu=\frac{2}{5}$ state for the Coulomb interaction in the lowest LL, and it becomes a MR GS with a large excitation gap in the first excited LL. The $N=10$ state at $2 l=21\left(\mathrm{a}^{\prime}-\mathrm{c}^{\prime}\right)$ is the Jain $\nu=\frac{2}{5}$ state in the $n=0 \mathrm{LL}$, and the MR state for $n=1$. Finally, the $N=10$ state at $2 l=23\left(\mathrm{a}^{\prime \prime}-\mathrm{c}^{\prime \prime}\right)$ contains four QE's in the Laughlin $\nu=\frac{1}{3}$ state in the $n=0 \mathrm{LL}$, and it is the $\nu=\frac{7}{3}$ state for $n=1$.

It can be seen in Fig. $1\left(\mathrm{a}-\mathrm{a}^{\prime \prime}\right)$ that for all three systems, the (Laughlin) correlations obtained for the $n=0$ Coulomb interaction are well reproduced by the model super-harmonic interaction $U_{x}$ with $x=0$ (the Laughlin correlations mean that the parentage $\mathcal{G}(1)$ from the $\mathcal{R}=1$ pair state is minimized). From Fig. 1 (b- $\left(\mathrm{b}^{\prime \prime}\right)$, the correlations in the $n=1 \mathrm{LL}$ are quite different, and they are better reproduced by the model interaction $U_{x}$ with $x=1$ (harmonic at short range). Clearly, the Laughlinlike "correlation hole" at $\mathcal{R}=1$ characteristic of low lying states in the $n=0 \mathrm{LL}$ is absent for $n=1$. Instead, the total parentage from the two states at $\mathcal{R}=1$ and 3 is minimized, which results in the shift of the maximum of

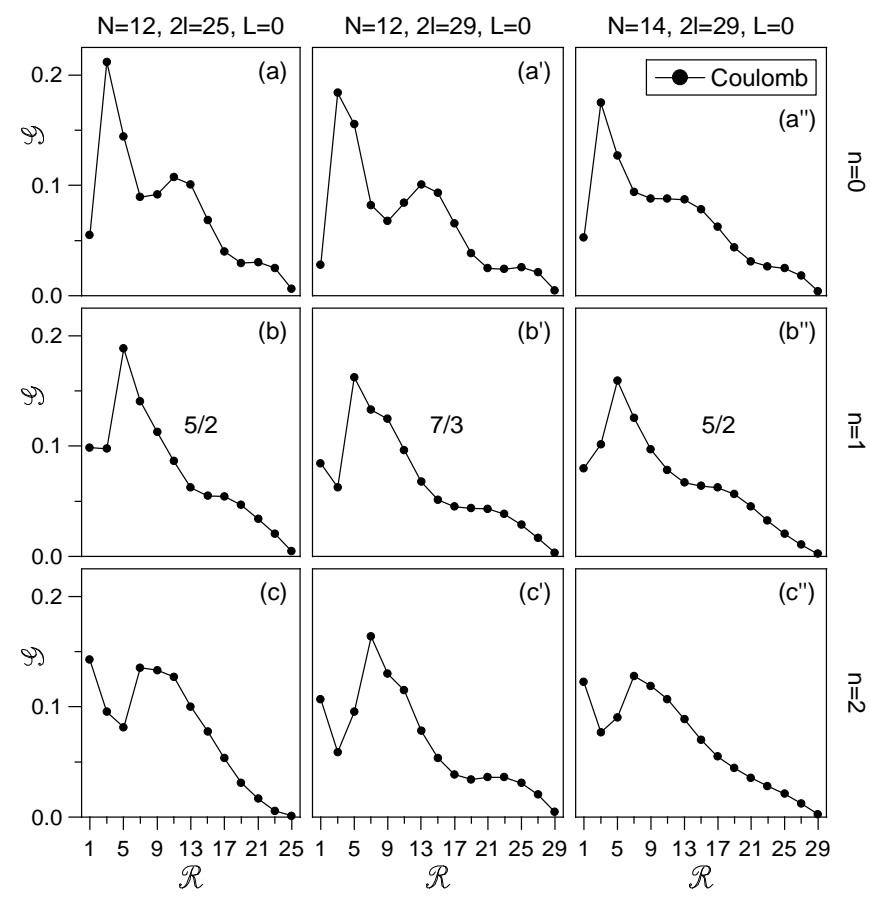

FIG. 5. The pair-correlation functions (coefficient of fractional parentage $\mathcal{G}$ vs. relative pair angular momentum $\mathcal{R}$ ) in the lowest energy $L=0$ state of $N$ electrons on a Haldane's sphere: $N=12$ and $2 l=25(\mathrm{a}-\mathrm{c}), N=12$ and $2 l=29$ $\left(\mathrm{a}^{\prime}-\mathrm{c}^{\prime}\right)$, and $N=14$ and $2 l=29\left(\mathrm{a}^{\prime \prime}-\mathrm{c}^{\prime \prime}\right)$, calculated for the Coulomb pseudopotential in the lowest $\left(\mathrm{a}-\mathrm{a}^{\prime \prime}\right)$, first excited $\left(\mathrm{b}-\mathrm{b}^{\prime \prime}\right)$, and second excited $\left(\mathrm{c}-\mathrm{c}^{\prime \prime}\right)$ Landau level.

$\mathcal{G}(\mathcal{R})$ from $\mathcal{R}=3$ (as is for $n=1$ ) to $\mathcal{R}=5$. Finally, the correlations for $n=2$ shown in Fig. $1\left(\mathrm{c}-\mathrm{c}^{\prime \prime}\right)$ are not well reproduced by $U_{x}$ with any value of $x$. A better approximation is obtained for a model pseudopotential $W_{x}(\mathcal{R})$ shown in Fig. 1(c), for which $W_{x}(1)=1, W_{x}(\mathcal{R} \geq 7)=0$, $W_{x}(3)=x \cdot V_{\mathrm{H}}(3)$, and $W_{x}(5)=x \cdot V_{\mathrm{H}}(5)$, that is $W_{x}(\mathcal{R})$ is harmonic between $\mathcal{R}=3$ and 7 , and $x$ controls harmonicity between $\mathcal{R}=1$ and 5 . Similar plots for larger systems of $N=12$ and 14 electrons interacting through Coulomb pseudopotentials are shown in Fig. 5. In the $n=1 \mathrm{LL}$, all three $L=0$ states in frames $\left(\mathrm{b}-\mathrm{b}^{\prime \prime}\right)$ are the incompressible ground states at $\nu=\frac{5}{2}$ or $\frac{7}{3}$.

Let us note that a tendency of $\mathcal{G}$ to decrease with increasing $\mathcal{R}$ ), observed most clearly at larger $\mathcal{R}$ (i.e., at separations beyond the correlation length), is characteristic of the closed (spherical) geometry. For example, $\mathcal{G}$ decreases linearly as a function of $\mathcal{R}$ ) for the $\nu=1$ state). However, the occurrence of minima and maxima in $\mathcal{G}(\mathcal{R})$, i.e. the differences between the values of $\mathcal{G}$ at neighboring values of $\mathcal{G}$, is independent of the geometry.

The above-described change of correlations when $n$ changes from 0 to 1 and 2 occurs for all low energy states (not only for the GS or the $L=0$ sector) and at any filling factor $\nu$ between about $\frac{1}{3}$ and $\frac{2}{3}$. Since the (Laughlin) correlation hole at small $\mathcal{R}$ results from the super-harmonicity of the pseudopotential at short range, 


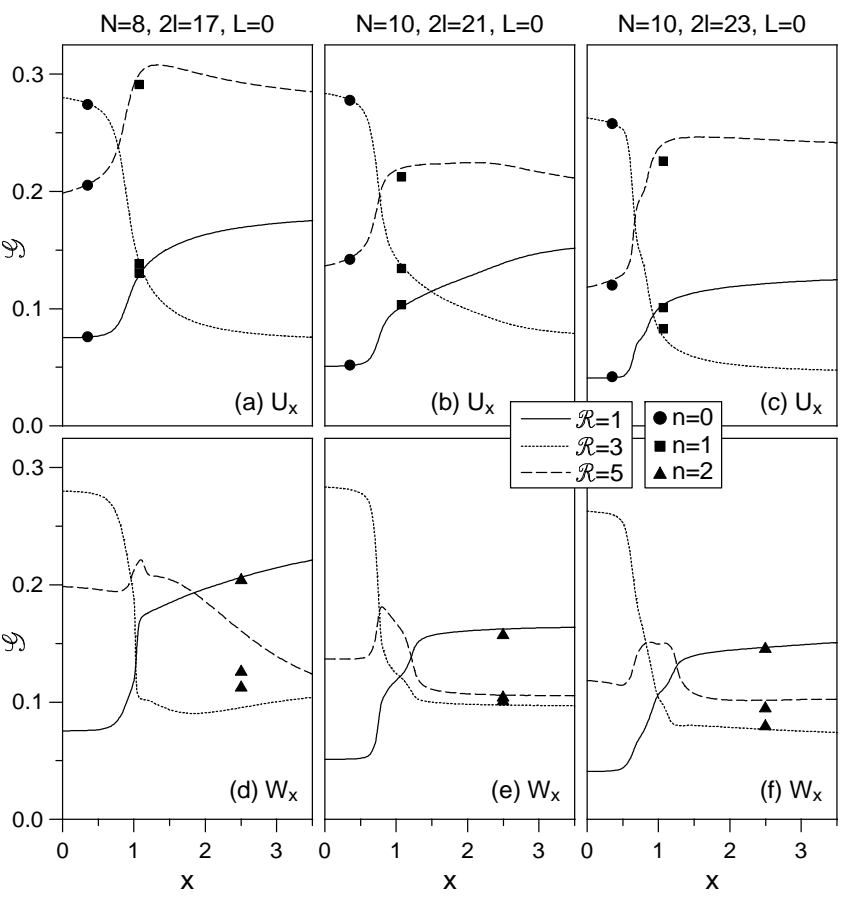

FIG. 6. The dependence of the coefficients of fractional parentage $\mathcal{G}$ from pair states at the smallest values of relative pair angular momentum, $\mathcal{R}=1,3$, and 5 , on the anharmonicity parameter $x$ of the model pseudopotentials $U_{x}$ (abc) and $W_{x}$ (def), calculated for the lowest $L=0$ state of $N$ electrons on a Haldane's sphere: $N=8$ and $2 l=17(\mathrm{ad}), N=10$ and $2 l=21$ (be), and $N=10$ and $2 l=23(\mathrm{cf})$. The values of $\mathcal{G}$ for the Coulomb pseudopotential in the lowest and two excited Landau levels are marked with symbols.

it is not surprising that this hole changes from a single pair state at $\mathcal{R}=1$ (for $n=0$ ) to a couple of pair states at $\mathcal{R}=1$ and 3 (for $n=1$ ) or at $\mathcal{R}=3$ and 5 (for $n=2$ ), when the range of $\mathcal{R}$ in which the (Coulomb) pseudopotential is sub-harmonic changes with $n$.

The crossover between the Laughlin correlations and pairing is best observed in the dependence of the CFGP's at a few smallest values of $\mathcal{R}$ on the anharmonicity parameter $x$ of the model interaction $U_{x}$. In Fig. 6 we show the plots of $\mathcal{G}(1), \mathcal{G}(3)$ and $\mathcal{G}(5)$ for the same lowest $L=0$ states as in Fig. 4, that is states of eight electrons at $2 l=17$ (a) and of ten electrons at $2 l=21$ (b) and 23 (c), obtained for the $U_{x}$ interaction. At $x<1$, when $U_{x}$ is super-harmonic in the entire range of $\mathcal{R}$, the Laughlin correlations occur, meaning that $\mathcal{G}(1)$ is close to its minimum possible value. As long as the interaction is super-harmonic (at short range), the values of CFGP's (and thus also the wavefunctions) weakly depend on the details of the pseudopotential (here, on $x$ ). At $x>1$, correlations of a different type occur, which persist up to the $x \rightarrow \infty$ limit. These correlations mean avoiding as much as possible the pair state at $\mathcal{R}=3$ (i.e., the most superharmonic part of $U_{x}$ ), which results in a large parentage from $\mathcal{R}=1$. The abrupt crossover between the two types of correlations occurs near $x=1$, where $\mathcal{G}(1)$ quickly increases from its minimum value, $\mathcal{G}(3)$ drops to its minimum value, and a maximum occurs in $\mathcal{G}(5)$. At the crossing points in frames (ab), $\mathcal{G}(1)$ is close to the value $(N-1)^{-1}$ describing $N_{2}=\frac{1}{2} N$ pairs each with $\mathcal{R}=1$. To obtain this value, which we will denote by $\mathcal{G}_{N_{2} \times 2}(1)$, we use the fact that the contribution of each $\nu=1$ droplet of $N^{\prime}$ electrons to the total number $\frac{1}{2} N(N-1) \mathcal{G}(1)$ of $\mathcal{R}=1$ pairs is $\frac{1}{2} N^{\prime}\left(N^{\prime}-1\right) \mathcal{G}_{1 \times N^{\prime}}(1)$, where the coefficient $\mathcal{G}_{1 \times N^{\prime}}(1)$ describes an isolated droplet.

The CFGP's calculated for the Coulomb pseudopotentials with $n=0$ and 1 are marked in Fig. 6 with full symbols. The symbols are plotted at arbitrary values of $x$ to show that the correlations for $V_{\mathrm{C}}^{(0)}$ can be well reproduced by $U_{x}$ with a finite $x<1$, and that the correlations for $V_{\mathrm{C}}^{(1)}$ are well approximated by $U_{x}$ with $x \approx 1$.

The most important conclusion from Fig. 6 is that the correlations in the partially filled (in particular, halffilled) LL are very sensitive to the harmonicity of the pseudopotential at short range, and the largest (smallest) number of pairs occurs at those of small values of $\mathcal{R}$, at which $V(\mathcal{R})$ is sub- (super-) harmonic. The Coulomb pseudopotential $V_{\mathrm{C}}^{(1)}$ in the $n=1 \mathrm{LL}$ is nearly harmonic between $\mathcal{R}=1$ and 5 , and thus the correlations it causes correspond to the crossover point between the sub- and super-harmonic regimes. The number of $\mathcal{R}=1$ pairs in the (MR) GS at $\nu=\frac{5}{2}$ is almost equal to half the number of electrons, $\frac{1}{2} N$. This is consistent with the notion of the paired character of the (MR) ground state, and supports its interpretation at the Laughlin paired $\nu_{2}^{*}=\frac{1}{4}$ state. The $\nu=\frac{7}{3}$ GS shown in Fig. 6 does not occur at the value of $2 l$ given by Eq. (5) or (6). Also, the value of $\mathcal{G}(1)$ in this state seems smaller than $\mathcal{G}_{N_{2} \times 2}(1)$. This precludes a description of this state as involving Laughlin correlations among $\frac{1}{2} N$ electron pairs each with $\mathcal{R}=1$.

The correlations induced by $V_{\mathrm{C}}^{(2)}$ are different from those in the $n=0$ or $n=1 \mathrm{LL}$ and cannot be modeled by $U_{x}$. The reason is that $V_{\mathrm{C}}^{(2)}$ is not super-harmonic up to $\mathcal{R}=7$. A better approximation is obtained using model pseudopotential $W_{x}(\mathcal{R})$. The plots of $\mathcal{G}(1), \mathcal{G}(3)$, and $\mathcal{G}(5)$ for the $W_{x}$ interaction in Fig. 6(def) show a similar break-up of Laughlin correlations at $x \approx 1$ as those for $U_{x}$. It is clear that the correlations in the $n=2 \mathrm{LL}$ can be modeled by $W_{x}$ with an appropriate $x>1$, and also that the effective value of $x$ (i.e., the correlations) depends on $\nu$. It can be expected that the tendency to occupy the $\mathcal{R}=1$ state and to avoid the $\mathcal{R}=3$ and 5 states will cause grouping of electrons into larger $\nu=1$ droplets. Indeed, the values of $\mathcal{G}(1)$ for the Coulomb states in Fig. 6(def) are much larger than $\mathcal{G}_{N_{2} \times 2}(1)$.

More insight into the nature of correlations in different LL's can be obtained from Figs. 7 and 8, in which we plot the dependences of the excitation gap $\Delta$ and parentage coefficients $\mathcal{G}(\mathcal{R})$ for a few smallest values of $\mathcal{R}$ on the value of $2 l$ (i.e., on $\nu$ ). The gaps $\Delta$ are taken from the $L=0$ GS's, and we set $\Delta=0$ when the GS has $L \neq 0$. 


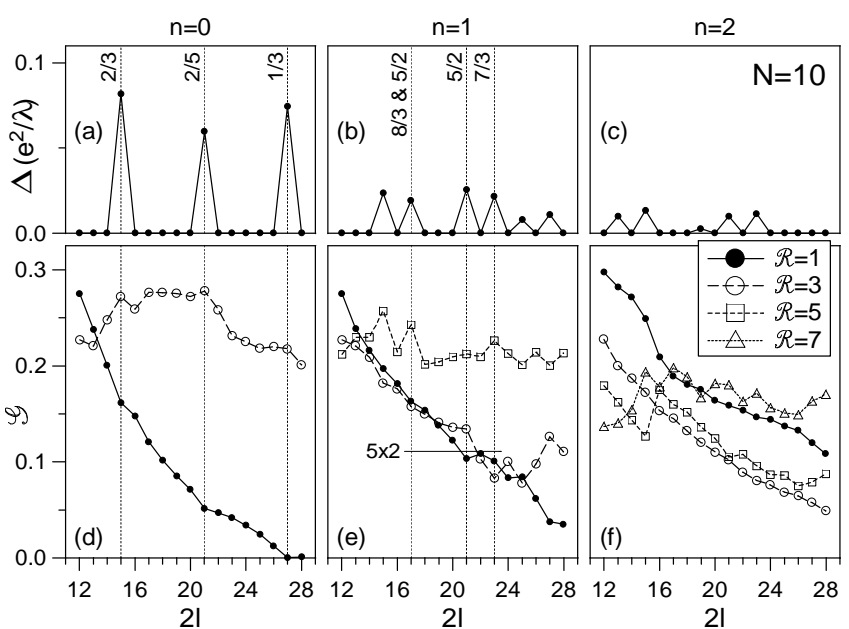

FIG. 7. The dependence of the excitation gap (abc) and the coefficients of fractional parentage $\mathcal{G}$ from pair states at the smallest values of the relative pair angular momentum, $\mathcal{R}=1,3,5$, and 7 (def) on $2 l$, calculated for the ground states of $N=10$ electrons on a Haldane's sphere, in the lowest (ad), first excited (be), and second excited (cf) Landau levels. For degenerate ground states $(L \neq 0)$ the gap is set to zero.

The CFGP's are calculated for the absolute GS's of $N$ electrons at given $2 l$ (not the lowest energy $L=0$ state).

The comparison of curves for $N=10$ and 12 confirms that to minimize total interaction energy at any $\nu$, electrons interacting through a pseudopotential $V(\mathcal{R})$ avoid as much as possible the total parentage from pairs states corresponding to $V_{\mathrm{AH}}(\mathcal{R})>0$. Because of relation (3), minimization of parentage from those most strongly repulsive pair states implies large parentage from less strongly repulsive pair states at the neighboring values of $\mathcal{R}$. Thus, for $n=0$ the occurrence of incompressible Laughlin-Jain states with large $\Delta$ coincides with downward peaks in $\mathcal{G}(1)$ and upward peaks in $\mathcal{G}(3)$. For $n=1$, where $\mathcal{G}(1)+\mathcal{G}(3)$ is minimized, large $\Delta$ coincides with upward peaks in $\mathcal{G}(5)$. Finally, for $n=2$ the occurrence of gaps seems to be connected with the behavior of $\mathcal{G}(7)$.

Note that in the $n=1 \mathrm{LL}$, the gap $\Delta=0.0049 e^{2} / \lambda$ in the $N=12$ electron system at $2 l=29$ is smaller than the gaps for $N \leq 11$ at the same filling factor (given by $2 l=3 N-7)$ and than the gap for $N=12$ at a neighboring $2 l=28$. The diminishing of $\Delta$ as a function of $N$ in the $2 l=3 N-7$ series of GS's indicates that this series might not describe the observed incompressible $\nu=\frac{7}{3}$ state in the $N \rightarrow \infty$ limit. In any case, it remains true that the occurrence of a finite-size $L=0$ GS with a large gap $\left(\Delta=0.0201 e^{2} / \lambda\right)$ at $N=12$ and $2 l=28$ coincides with an upward cusp in $\mathcal{G}(5)$.

The occurrence of similar maxima in $\mathcal{G}(5)$ at $\nu=\frac{5}{2}$, $\frac{7}{3}$, and $\frac{8}{3}$ (or, more exactly, at the values of $N$ and $2 l$ at which non-degenerate GS's with large gaps occur) for $n=1$ indicates common correlations in these three states, different from those in other LL's. We have marked the values of $\mathcal{G}(1)$ corresponding to grouping of $N$

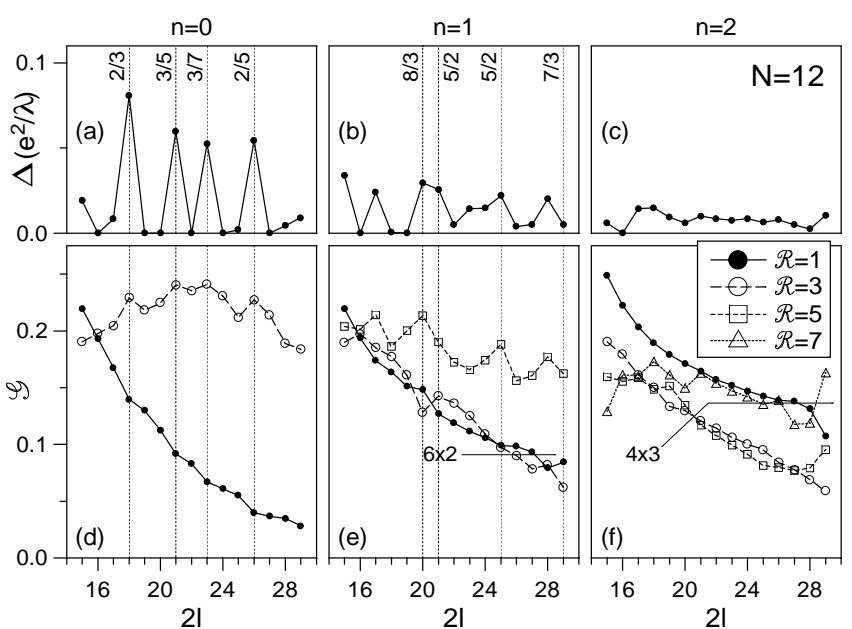

FIG. 8. The dependence of the excitation gap (abc) and the coefficients of fractional parentage $\mathcal{G}$ from pair states at the smallest values of the relative pair angular momentum, $\mathcal{R}=1,3,5$, and 7 (def) on $2 l$, calculated for the ground states of $N=12$ electrons on a Haldane's sphere, in the lowest (ad), first excited (be), and second excited (cf) Landau levels. For degenerate ground states $(L \neq 0)$ the gap is set to zero.

electrons into $\frac{1}{2} N$ pairs, $\mathcal{G}_{N_{2} \times 2}(1)=(N-1)^{-1}$. Clearly, the average number of $\mathcal{R}=1$ pairs decreases with increasing $2 l$ which seems to disagree with the prediction of Laughlin paired $\nu_{2}^{*}=\left(2 q_{2}\right)^{-1}$ states for all values of $q_{2}$ between 1 and 4 (for Laughlin paired states one should expect $\mathcal{G}(1) \approx(N-1)^{-1}$ independently of $\left.2 l\right)$. However, the number of $\mathcal{R}=1$ pairs is roughly equal to $\frac{1}{2} N$ for $2 l$ corresponding to the MR state at $\nu=\frac{5}{2}$, which suggests the Laughlin paired $\nu_{2}^{*}=\frac{1}{4}$ state as an appropriate description at this particular filling.

The observation that $\mathcal{G}(1)$ in the $n=1$ LL decreases monotonically as a function of $2 l$ and that $\mathcal{G}(1) \approx(N-$ $1)^{-1}$ at $\nu=\frac{5}{2}$ suggests that all $N$ electrons form pairs at exactly $\nu=\frac{5}{2}$, but only a fraction of electrons pair up $\left(N_{2}<\frac{1}{2} N\right.$ and $\left.N_{1}>0\right)$ when $\nu<\frac{5}{2}$, and some pairs are replaced by larger $\nu=1$ clusters (e.g., into threeelectron droplets each with $\left.l_{3}=3 l-3\right)$ when $\nu>\frac{5}{2}$. The break-up or clustering of pairs can be understood from the behavior of the effective pseudopotentials describing interaction between electrons, pairs, and larger drpplets, and will be discussed in a subsequent publication 39

In the $n=2 \mathrm{LL}$, the average number of $\mathcal{R}=1$ pairs is larger than $\frac{1}{2} N$ indicating formation of larger $\nu=1$ droplets (stripes 33.34 ) separated from one another. As marked in Fig. 8(f), in the (fairly small) $N=12$ electron system, $\mathcal{G}(1) \approx \mathcal{G}_{4 \times 3}(1)=\frac{3}{2}(N-1)^{-1}$ near the halffilling, which corresponds to four three-electron droplets.

\section{CONCLUSION}

Using exact numerical diagonalization in Haldane's spherical geometry we have studied electron correlations 
near the half-filling of the lowest and excited LL's. We have shown that the electrons interacting through a pseudopotential $V(\mathcal{R})$ generally avoid pairs states corresponding to large and positive anharmonicity of $V(\mathcal{R})$. We have shown that as a result of different behavior of $V(\mathcal{R})$ in different LL's, the correlations in the excited LL's are different than the Laughlin correlations in the lowest LL. This confirms different origin of the incompressibility of the $\nu=\frac{1}{3}$ and $\frac{7}{3}$ GS's. In particular, correlations in the partially filled first excited $(n=1)$ LL depend critically on the harmonic behavior of the Coulomb pseudopotential at short range, and are destroyed when the pseudopotential becomes either strongly super-harmonic (as for $n=0$ ) or strongly subharmonic (as for $n=2$ ). The Moore-Read incompressible state at $\nu=\frac{5}{2}$ occurs at the LL degeneracy (flux) given by $2 l=2 N-3$ (and $2 l=2 N+1$ for its particle-hole conjugate). This value of $2 l$ and the calculated CFGP's for the low lying states indicate that the Moore-Read $\nu=\frac{5}{2}$ state can be understood as a Laughlin correlated $\nu_{2}^{*}=\frac{1}{4}$ bosonic state of electron pairs. Although other filling factors at which incompressibility is observed in the $n=1 \mathrm{LL}\left(\nu=\frac{7}{3}\right.$ and $\left.\frac{8}{3}\right)$ also arise in the sequence of Laughlin paired $\nu_{2}^{*}=\left(2 q_{2}\right)^{-1}$ states, we find no evidence that these are the actual Coulomb GS's. The two series of finite-size non-degenerate GS's that we find in our numerical calculations and that extrapolate to $\nu=\frac{7}{3}$ and $\frac{8}{3}$ for $N \rightarrow \infty$ occur at $2 l=3 N-7$ and $\frac{3}{2} N+2$. These values of $2 l$ are different from both these of Laughlin-Jain GS's at $\nu=\frac{1}{3}$ and $\frac{2}{3}$ in the $n=0 \mathrm{LL}$, and those of the hypothetical Laughlin paired states at $\nu_{2}^{*}=\frac{1}{8}$ and $\frac{1}{2}$.

\section{ACKNOWLEDGMENT}

The author gratefully acknowledges helpful discussions with John J. Quinn (Univ. Tennessee), partial support of Grant DE-FG02-97ER45657 from the Materials Science Program - Basic Energy Sciences of the US Department of Energy, and of Grant 2P03B11118 from the Polish Sci. Comm., and thanks the Joint Institute for Computational Science at the Univ. Tennessee for providing access to the IBM SP2 supercomputer and for user support.

${ }^{1}$ R. Laughlin, Phys. Rev. Lett. 50, 1395 (1983).

${ }^{2}$ D. C. Tsui, H. L. Störmer, and A. C. Gossard, Phys. Rev. Lett. 48, 1559 (1982).

${ }^{3}$ The Quantum Hall Effect, edited by R. E. Prange and S. M. Girvin (Springer-Verlag, New York, 1987).

${ }^{4}$ H. L. Störmer, D. C. Tsui, and A. C. Gossard, Rev. Mod. Phys. 71, S298 (1999).
${ }^{5}$ F. D. M. Haldane and E. H. Rezayi, Phys. Rev. Lett. 60, 956 (1988).

${ }^{6}$ A. Wójs and J. J. Quinn, Phil. Mag. B 80, 1405 (2000).

${ }^{7}$ J. J. Quinn and A. Wójs, J. Phys.: Cond. Mat. 12, R265 (2000).

${ }^{8}$ F. D. M. Haldane, Phys. Rev. Lett. 51, 605 (1983).

${ }^{9}$ R. B. Laughlin, Surf. Sci. 142, 163 (1984).

10 B. I. Halperin, Phys. Rev. Lett. 52, 1583 (1984).

${ }^{11}$ P. Sitko, K.-S. Yi, and J. J. Quinn, Phys. Rev. B 56, 12417 (1997).

12 A. Wójs and J. J. Quinn, Phys. Rev. B 61, 2846 (2000).

13 J. K. Jain, Phys. Rev. Lett. 63, 199 (1989).

14 A. Lopez and E. Fradkin, Phys. Rev. B 44, 5246 (1991).

15 J. K. Jain and V. J. Goldman, Phys. Rev. B 45, 1255 (1992).

16 B. I. Halperin, P. A. Lee, and N. Read, Phys. Rev. B 47, 7312 (1993).

${ }^{17}$ R. L. Willet, J. P. Eisenstein, H. L. Störmer, D. C. Tsui, A. C. Gossard, and J. H. English, Phys. Rev. Lett. 59, 1776 (1987).

18 J. P. Eisenstein, in Perspectives in Quantum Hall Effects, edited by S. Das Sarma and A. Pinczuk (WileyInterscience, New York, 1996), p. 37.

19 J. P. Eisenstein, R. L. Willet, H. L. Störmer, D. C. Tsui, A. C. Gossard, and J. H. English, Phys. Rev. Lett. 61, 997 (1988).

20 J. P. Eisenstein, H. L. Störmer, L. Pfeiffer, and K. W. West, Phys. Rev. Lett. 62, 1540 (1989).

${ }^{21}$ J. P. Eisenstein, R. L. Willet, H. L. Störmer, L. Pfeiffer, and K. W. West, Surf. Sci. 229, 31 (1990).

${ }^{22}$ R. H. Morf, Phys. Rev. Lett. 80, 1505 (1998).

${ }^{23}$ L. Belkhir and J. K. Jain, Phys. Rev. Lett. 70, 643 (1993).

${ }^{24}$ K. Park, V. Melik-Alaverdian, N. E. Bonesteel, and J. K. Jain, Phys. Rev. B 58, R10 167 (1998).

${ }^{25}$ B. I. Halperin, Helv. Phys. Acta 56, 75 (1983).

${ }^{26}$ R. H. Morf, N. d'Ambrumenil, and B. I. Halperin, Phys. Rev. B 34, 3037 (1986).

${ }^{27}$ G. Moore and N. Read, Nucl. Phys. B 360, 362 (1991).

${ }^{28}$ M. Greiter, X.-G. Wen, and F. Wilczek, Phys. Rev. Lett. 66, 3205 (1991).

${ }^{29}$ E. H. Rezayi and F. D. M. Haldane, Phys. Rev. Lett. 84, 4685 (2000).

${ }^{30}$ A. de Shalit and I. Talmi, Nuclear Shell Theory (Academic Press, New York and London, 1963).

${ }^{31}$ R. D. Cowan, The Theory of Atomic Structure and Spectra (University of California Press, Berkeley, Los Angeles, and London, 1981).

32 A. H. MacDonald and S. M. Girvin, Phys. Rev. B, 33, 4009 (1986).

33 A. A. Koulakov, M. M. Fogler, and B. I. Shklovskii, Phys. Rev. Lett. 76, 499 (1996).

${ }^{34}$ E. H. Rezayi, F. D. M. Haldane, and K. Yang, Phys. Rev. Lett. 83, 1219 (1999).

35 T. T. Wu and C. N. Yang, Nucl. Phys. B 107, 365 (1976); Phys. Rev. D 16, 1018 (1977).

${ }^{36}$ G. Fano, F. Ortolani, and E. Colombo, Phys. Rev. B 34, 2670 (1986).

37 A. Wójs, I. Szlufarska, K. S. Yi, and J. J. Quinn, Phys. Rev. B 60, R11 273 (1999).

${ }^{38}$ D. C. Marinescu, J. J. Quinn, P. Sitko, and K. S. Yi, Phys. 
Rev. B 56, 14941 (1997).

${ }^{39}$ A. Wójs and J. J. Quinn, to be published. 